\title{
Clinical Manifestations and Outcomes in Disease-Modifying Antirheumatic Drug-Naive Adult Patients with Chronic Chikungunya Arthritis
}

\author{
Nicolle Medina-Cintrón, ${ }^{1}$ Idali Martínez, ${ }^{2}$ Naydi Pérez-Ríos, ${ }^{3}$ Yaritza Berríos-López, ${ }^{1}$ and Luis M. Vilá ${ }^{1 *}$ \\ ${ }^{1}$ Division of Rheumatology, University of Puerto Rico Medical Sciences Campus, San Juan, Puerto Rico; ${ }^{2}$ Department of Microbiology and Medical \\ Zoology, University of Puerto Rico Medical Sciences Campus, San Juan, Puerto Rico; ${ }^{3}$ Hispanic Alliance for Clinical and Translational Research,
} University of Puerto Rico Medical Sciences Campus, San Juan, Puerto Rico

\begin{abstract}
Most studies on chronic chikungunya virus (CHIKV) arthritis include patients treated with disease-modifying antirheumatic drugs (DMARDs), likely altering the expression of clinical manifestations and outcome. Therefore, we sought to evaluate the clinical features and correlates in DMARD-naive patients with chronic CHIKV arthritis. We conducted a case-control study in adult patients with serologically confirmed CHIKV infection in Puerto Rico. Demographic features, clinical manifestations, comorbidities, disease activity (per Clinical Disease Activity Index [CDAI]), functional status (per Health Assessment Questionnaire Disability Index [HAQ-DI]), and pharmacologic treatment were ascertained. Patients with and without chronic CHIKV arthritis were compared. Furthermore, a sub-analysis was performed among patients with chronic CHIKV who presented with mild disease activity versus moderate-to-high disease activity at study visit. In total, 61 patients were studied; 33 patients had chronic arthritis and 28 had resolved arthritis. Patients with chronic arthritis had significantly more diabetes mellitus, chronic back pain, and fever, tiredness, and myalgias on the acute phase. The mean (SD) HAQ score was 0.95 (0.56), and $57.6 \%$ had moderate-to-high disease activity. Patients with moderate-to-high disease activity had higher scores in overall HAQ-DI and HAQ-DI categories (dressing and grooming, arising, hygiene, reaching, and activities) than in those with mild activity. In conclusion, in this group of DMARD-naive patients with chronic CHIKV arthritis, nearly $58 \%$ had moderate-to-high disease activity and had substantial functional disability. Diabetes mellitus, chronic back pain, and some manifestations on acute infection were associated with chronic CHIKV arthritis.
\end{abstract}

\section{INTRODUCTION}

Chikungunya virus (CHIKV) is a single-stranded RNA virus from the Alphavirus genus transmitted to humans through the Aedes aegypti and Aedes albopictus mosquitoes. ${ }^{1,2}$ It was first described in 1955 by Robinson after an outbreak in East Africa in $1952 .^{3}$ By 2013, it reached the Western Hemisphere, accumulating more than 3 million cases in the Americas. ${ }^{4}$ In Puerto Rico, the first locally acquired CHIKV infection was documented in May $2014 .^{5}$

Acute CHIKV infection is characterized by high-grade fever, polyarthralgia, polyarthritis, maculopapular rash, fatigue, and myalgias. ${ }^{1,2}$ Viremia usually lasts from 5 to 10 days. Affected individuals may develop CHIKV manifestations lasting months to years after infection that vary from $25 \%$ to $80 \%$ of patients. $^{6-16}$ Risk factors for developing chronic CHIKV manifestations include female gender, older age, comorbid conditions (arterial hypertension, osteoarthritis, and diabetes mellitus), and severe disease in the acute phase. , $^{2,9,13-16}$

Patients with chronic CHIKV arthritis have polyarthralgia and/or polyarthritis affecting both small and large joints, on occasions mimicking autoimmune inflammatory arthritis such as rheumatoid arthritis (RA). ${ }^{1,2,17}$ It is associated with significant disability and loss of productivity. ${ }^{13-15,18-20}$ Nonsteroidal anti-inflammatory drugs (NSAIDs), low-dose corticosteroids, and synthetic and biologic disease-modifying antirheumatic drugs (DMARDs) have been used for chronic CHIKV arthritis. ${ }^{4,21}$ However, recommendations have a low level of evidence due to a lack of randomized clinical trials. As climate shifts, it is expected that more individuals will become at risk of

\footnotetext{
*Address correspondence to Luis M. Vilá, Division of Rheumatology, University of Puerto Rico Medical Sciences Campus, P.O. Box 365067, San Juan PR 00936-5067, Puerto Rico. E-mail: luis.vila2@
} upr.edu infection. ${ }^{2}$ Without effective vaccines or antiviral therapies, consequences of CHIKV infection sequelae may remain a substantial burden for public health.

Despite limited evidence for therapy, most studies describing patients with chronic CHIKV arthritis include individuals treated with DMARDs. As expected, the use of these drugs alters the expression of clinical manifestations during the chronic phase. Understanding the natural history of a disease allows refocusing research priorities for prevention, treatment, and recovery. Therefore, we sought to evaluate the clinical features and correlates in patients with chronic CHIKV arthritis who have not received therapy with DMARDs.

\section{MATERIALS AND METHODS}

Patient population. We conducted a case-control study in CHIKV-infected adults with and without chronic arthralgia and/or arthritis. Study participants included adults ( $\geq 21$ years) who had CHIKV infection. All patients had inflammatory polyarthritis in the acute phase of infection defined as the period $<3$ months from infection. Previous CHIKV infection was confirmed by viral IgG detection using ELISA. Exclusion criteria included the following: 1) minors, $<21$ years of age; 2) patients having or fulfilling classification criteria for autoimmune/ inflammatory rheumatic diseases such as RA, seronegative arthritis, and systemic lupus erythematosus, among others; 3) patients treated with corticosteroids 3 months after the onset of CHIKV; 4) those treated at any time with synthetic or biologic DMARDs; 5) patients who were unwilling to give informed consent; and 6) those unable to understand the informed consent form and the protocol procedure.

Chronic CHIKV arthritis was defined as arthralgias/arthritis lasting $\geq 3$ months after disease onset. To have patients with chronic CHIKV arthritis with at least 3 months of follow-up, we included those who had acute CHIKV infection $\geq 6$ months 
before enrollment. Controls were defined as individuals who had CHIKV infection but recovered completely in less than 3 months after infection. Matching by age and gender was used for the selection of the control participants. Participants were enrolled between February 2016 and April 2018 at the University of Puerto Rico Medical Sciences Campus (UPRMSC), San Juan, Puerto Rico, and the Ponce Health Sciences University, Ponce, Puerto Rico. At study visit, a comprehensive history and physical examination were performed to each participant by a rheumatologist certified in Internal Medicine and Rheumatology by the American Board of Internal Medicine. This study was approved by the UPR-MSC Institutional Review Board (number 2460215). Research on human subjects was performed in compliance with the Helsinki Declaration. Before study participation, each subject signed an informed consent.

Variables. Sociodemographic features, lifestyle behaviors, clinical manifestations (on acute CHIKV infection and at study visit), cumulative comorbidities, pharmacologic treatment, disease activity, and functional status were ascertained. Sociodemographic features included age, gender, time period from acute infection to study visit, and years of education. Among lifestyle behaviors, cigarette smoking and exercise were determined. The following clinical manifestations were ascertained on the acute infectious phase: arthralgias, arthritis, myalgias, morning stiffness, fever, tiredness, and maculopapular rash. Distribution of joint tenderness and joint swelling at study visit were examined. Cumulative comorbidities included those that have been linked with chronic CHIKV arthritis: overweight/obesity per body mass index, arterial hypertension, diabetes mellitus (type 1 or type 2), chronic back pain, and osteoarthritis. Exposure to acetaminophen, NSAIDs, and prednisone (or equivalent) on the acute phase of infection was determined. Disease activity was measured using the Clinical Disease Activity Index (CDAl), which consists of a 28 tender and 28 swollen joint count plus patient's and evaluator's global assessments of general health using a visual analog scale. ${ }^{22}$ Clinical Disease Activity Index (CDAI) scores of 0.0-2.8, 2.9-10.0, 10.1-22.0, and 22.1-76.0 correspond to clinical remission, mild activity, moderate activity, and high activity, respectively. Functional status was determined using the Health Assessment Questionnaire Disability Index (HAQ-DI), which evaluates the functional disability in eight categories of regular daily activities. Health Assessment Questionnaire Disability Index scores of 0, 1, 2, and 3 are interpreted as no disability, moderate disability, severe disability, and complete disability, respectively. ${ }^{23}$

Statistical analysis. Patients with and without chronic CHIKV arthritis were compared in terms of their sociodemographic features, lifestyle behaviors, clinical manifestations (on acute CHIKV infection and at study visit), cumulative comorbidities, pharmacologic treatment, disease activity, and functional status characteristics. Comparisons between groups (with and without chronic CHIKV arthritis) were performed using Pearson's chi-squared test or Fisher's exact test as appropriate for categorical variables. For continuous variables, Shapiro-Wilk and Bartlett's tests were performed to evaluate normality and homoscedasticity of the data. A Wilcoxon rank-sum test was later chosen to compare continuous variables by CHIKV groups. Similar tests (e.g., Fisher's exact test, Pearson's chi-squared test, and the Wilcoxon rank-sum test) were performed for a sub-analysis among patients with chronic CHIKV arthritis who presented with mild disease activity $(\mathrm{CDAl}<10)$ versus moderate-to-high disease activity $(C D A I \geq 10)$ at study visit. $P$-values $\leq 0.05$ were considered statistically significant. All statistical analyses were performed using STATA SE 16 (Stata Corp., College Station, TX).

\section{RESULTS}

Seventy-eight patients who had CHIKV infection were screened. Seventeen subjects were excluded $(R A=2$, psoriatic arthritis $=1$, systemic lupus erythematosus $=3$, fibromyalgia syndrome $=2$, chronic therapy with low-dose prednisone $=5$, conventional DMARD therapy $=3$, and biologic DMARD therapy =1). In total, 61 patients were studied; $41(67.2 \%)$ were women. The mean (SD) period between onset of CHIKV infection and study visit was 23.4 (9.4) months. Thirty-three patients had chronic CHIKV arthritis, and 28 had resolved arthritis.

Table 1 shows sociodemographic features, lifestyle behaviors, cumulative comorbidities, clinical manifestations, and treatment on acute infection between patients, disease activity, and functional status with and without chronic CHIKV arthritis. Those with chronic CHIKV arthritis were less likely to exercise $(21.2 \%$ versus $57.1 \%, P=0.004)$ and had more diabetes mellitus (18.2\% versus $0.0 \%, P=0.027)$ and chronic back pain $(24.2 \%$ versus $0.0 \%, P=0.006)$ than those with resolved arthritis. Patients who presented with myalgias (97.0\% versus $75.0 \%, P=0.019)$, fever $(97.0 \%$ versus $78.6 \%$, $P=0.041)$, and tiredness $(100 \%$ versus $82.1 \%, P=0.017)$ on acute infection were more likely to develop chronic CHIKV arthritis. Those with chronic CHIKV arthritis were treated more frequently with NSAIDs $(75.8 \%$ versus $50.0 \%, P=0.037)$ during the acute phase. As expected, mean (SD) CDAI (14.0 [9.3] versus 0.07 [0.3], $P=<0.001)$ and HAQ-DI (0.95 [0.56] versus 0.06 [0.17], $P=<0.001$ ) scores were higher for patients with chronic CHIKV arthritis at study visit. No significant differences were found for age, gender, time period from acute infection to study visit, years of education, smoking, overweight/obesity, arterial hypertension, dyslipidemia, and osteoarthritis.

Table 2 shows the distribution of joint tenderness and joint swelling among patients with chronic CHIKV arthritis at study visit. Overall, joints of upper extremities were more commonly involved than those of lower extremities. Joint tenderness was most common in metacarpophalangeal (MCP) joints (54.6\%), shoulders $(51.5 \%)$, proximal interphalangeal (PIP) joints (45.5\%), and knees (39.4\%). Joint swelling was more commonly observed in PIP joints (15.2\%), MCP joints (12.1\%), shoulders (9.1\%), wrists $(6.1 \%)$, and ankles $(6.1 \%)$.

Nineteen (57.6\%) patients with chronic CHIKV arthritis had moderate-to-high disease activity. Table 3 shows a sub-analysis between patients with chronic CHIKV arthritis presenting with mild disease activity $(\mathrm{CDAl} \leq 10)$ and moderateto-high disease activity (CDAI > 10). Those with moderate-tohigh activity had lower mean (SD) years of education (15.6 [4.5] versus 17.4 [2.7], $P=0.049$ ) and a higher mean (SD) HAQ score (1.19 [0.47] versus 0.63 [0.50], $P=0.006$ ) than those with mild disease activity. Among HAQ-DI categories, patients with moderate-to-high disease activity presented with more functional disability in the following categories: dressing and grooming, arising, hygiene, reaching, and usual activities. No differences were found for demographic features, lifestyle 
TABLE 1

Demographic features, lifestyle behaviors, comorbidities, clinical manifestations, disease activity, and functional status in patients with and without chronic CHIKV arthritis

\begin{tabular}{|c|c|c|c|}
\hline Features & Chronic CHIKV arthritis $(n=33)$ & Resolved CHIKV arthritis $(n=28)$ & $P$-value \\
\hline Mean age (SD) (years) & $52.5(10.5)$ & $48.3(13.7)$ & 0.205 \\
\hline Gender, \%women & 69.7 & 64.3 & 0.786 \\
\hline $\begin{array}{l}\text { Mean period from acute infection to study } \\
\text { visit (SD) (months) }\end{array}$ & $25.3(10.8)$ & $21.1(7.0)$ & 0.651 \\
\hline Mean years of education (SD) & $16.3(3.9)$ & $17.0(3.9)$ & 0.631 \\
\hline \multicolumn{4}{|l|}{ Lifestyle behaviors, \% } \\
\hline Smoking & 12.1 & 14.3 & $>0.999$ \\
\hline Exercise & 21.2 & 57.1 & 0.004 \\
\hline \multicolumn{4}{|l|}{ Cumulative comorbidities, $\%$} \\
\hline Overweight/obese (body mass index $\geq 25$ ) & 87.9 & 78.6 & 0.490 \\
\hline Arterial hypertension & 24.2 & 28.6 & 0.775 \\
\hline Diabetes mellitus (type 1 or type 2 ) & 18.2 & 0.0 & 0.027 \\
\hline Dyslipidemia & 18.2 & 25.0 & 0.517 \\
\hline Chronic back pain & 24.2 & 0.0 & 0.006 \\
\hline Osteoarthritis & 15.2 & 14.3 & $>0.999$ \\
\hline \multicolumn{4}{|l|}{ Clinical manifestations on acute infection, \% } \\
\hline Arthralgias & 100 & 96.4 & 0.459 \\
\hline Arthritis & 87.9 & 71.4 & 0.107 \\
\hline Myalgias & 97.0 & 75.0 & 0.019 \\
\hline Morning stiffness & 97.0 & 82.1 & 0.085 \\
\hline Fever & 97.0 & 78.6 & 0.041 \\
\hline Tiredness & 100 & 82.1 & 0.017 \\
\hline Maculopapular rash & 93.9 & 75.0 & 0.067 \\
\hline \multicolumn{4}{|l|}{ Treatment on acute infection, $\%$} \\
\hline Acetaminophen & 90.9 & 85.7 & 0.693 \\
\hline Nonsteroidal anti-inflammatory drugs & 75.8 & 50.0 & 0.037 \\
\hline Prednisone & 33.3 & 14.3 & 0.085 \\
\hline $\begin{array}{l}\text { Clinical Disease Activity Index mean } \\
\text { score (SD) at study visit }\end{array}$ & $14.0(9.3)$ & $0.07(0.3)$ & $<0.001$ \\
\hline $\begin{array}{l}\text { Health Assessment Questionnaire } \\
\text { Disability Index mean score (SD) at } \\
\text { study visit }\end{array}$ & $0.95(0.56)$ & $0.06(0.17)$ & $<0.001$ \\
\hline
\end{tabular}

behaviors, comorbidities, and clinical manifestations and treatment on acute infection.

\section{DISCUSSION}

Patients with chronic CHIKV arthritis have similar clinical manifestations, disease activity, and functional disability when compared with those with autoimmune inflammatory arthritis such as RA. Previous studies have not evaluated clinical features and correlates in DMARD-naive patients with chronic CHIKV arthritis. This approach may allow studying the natural history of chronic CHIKV arthritis. Thus, we examined the clinical features in patients with serologically confirmed CHIKV infection who did not receive therapy with DMARDs and found that chronic arthritis was associated with diabetes mellitus, chronic back pain, and several symptoms on the acute phase of infection. Among patients with chronic CHIKV arthritis, joints of upper extremities were more commonly involved, more than half had moderate to high disease activity, and most presented moderate functional disability.

We found an association of chronic CHIKV arthritis with diabetes mellitus. Similarly, in patients from La Réunion Island, diabetes mellitus was a risk factor for chronic CHIKV arthritis. $^{24}$ This association is not unexpected, as patients with diabetes mellitus exhibit inflammatory features. For example, diabetes mellitus patients have elevated IL- 6 and IL- $1 \beta$, which seem to be critical in the pathogenesis of chronic CHIKV arthritis. ${ }^{25}$ High levels of these cytokines are observed in patients with severe acute CHIKV infection and persistent arthralgias. $^{26,27}$ Unlike what was reported by Sissoko et al., ${ }^{28}$ we did not find an association with osteoarthritis in patients

TABLE 2

Joint tenderness and swelling in patients with chronic chikungunya virus arthritis

\begin{tabular}{lccr}
\hline \multicolumn{1}{c}{ Joints } & Joint tenderness, $n(\%)$ & Joint swelling, $n(\%)$ & Joint tenderness or swelling, $n$ (\%) \\
\hline Proximal interphalangeal joints & $15(45.5)$ & $5(15.2)$ & $16(48.5)$ \\
Metacarpophalangeal joints & $18(54.6)$ & $4(12.1)$ & $18(54.6)$ \\
Wrists & $12(36.4)$ & $2(6.1)$ & $12(36.4)$ \\
Elbows & $12(36.4)$ & $0(0.0)$ & $12(36.4)$ \\
Shoulders & $17(51.5)$ & $3(9.1)$ & $17(51.5)$ \\
Knees & $13(39.4)$ & $0(0.0)$ & $13(39.4)$ \\
Ankles & $7(21.2)$ & $2(6.1)$ & $7(21.2)$ \\
Metatarsophalangeal joints & $6(18.2)$ & $1(3.0)$ & $6(18.2)$ \\
\hline
\end{tabular}


TABLE 3

Demographic features, lifestyle behaviors, comorbidities, clinical manifestations on acute infection and at study visit, and functional status in patients with chronic CHIKV presenting with mild and moderate-to-high disease activity per Clinical Disease Activity Index

\begin{tabular}{|c|c|c|c|c|}
\hline Features & All chronic $\mathrm{CHIKV}$ arthritis $(n=33)$ & Mild disease activity $(n=14)$ & Moderate-to-high disease activity $(n=19)$ & $P$-value \\
\hline Mean age (SD) (years) & $52.5(10.5)$ & $51.7(11.5)$ & $53.1(10.0)$ & 0.771 \\
\hline Gender, \% women & 69.7 & 85.7 & 57.9 & 0.131 \\
\hline $\begin{array}{l}\text { Mean period from acute infection to study } \\
\text { visit (SD) (months) }\end{array}$ & $25.3(10.8)$ & $23.0(10.7)$ & $27.1(10.8)$ & 0.084 \\
\hline Mean years of education (SD) & $16.3(3.9)$ & $17.4(2.7)$ & $15.6(4.5)$ & 0.049 \\
\hline \multicolumn{5}{|l|}{ Lifestyle behaviors, \% } \\
\hline Smoking & 12.1 & 0.0 & 21.1 & 0.119 \\
\hline Exercise & 21.2 & 21.4 & 21.1 & $>0.999$ \\
\hline \multicolumn{5}{|l|}{ Comorbidities, \% } \\
\hline Overweight/obese (body mass index $\geq 25$ ) & 87.9 & 85.7 & 89.5 & $>0.999$ \\
\hline Arterial hypertension & 24.2 & 37.5 & 62.5 & $>0.999$ \\
\hline Diabetes mellitus & 18.2 & 14.3 & 21.1 & $>0.999$ \\
\hline Dyslipidemia & 18.2 & 28.6 & 10.5 & 0.363 \\
\hline Chronic back pain & 24.2 & 28.6 & 21.1 & 0.695 \\
\hline Osteoarthritis & 15.2 & 14.3 & 15.8 & $>0.999$ \\
\hline \multicolumn{5}{|l|}{ Clinical manifestations on acute infection, \% } \\
\hline Arthralgia & 100 & 100 & 100 & - \\
\hline Arthritis & 87.9 & 78.6 & 94.7 & 0.288 \\
\hline Myalgias & 97.0 & 92.9 & 100 & 0.424 \\
\hline Morning stiffness & 97.0 & 92.9 & 100 & 0.424 \\
\hline Fever & 97.0 & 92.9 & 100 & 0.424 \\
\hline Tiredness & 100 & 100 & 100 & - \\
\hline Maculopapular rash & 93.9 & 100 & 89.5 & 0.496 \\
\hline \multicolumn{5}{|l|}{ Treatment on acute infection, $\%$} \\
\hline Acetaminophen & 90.9 & 92.9 & 89.5 & $>0.999$ \\
\hline Nonsteroidal anti-inflammatory drugs & 75.8 & 85.7 & 68.4 & 0.416 \\
\hline Prednisone & 33.3 & 35.7 & 31.6 & $>0.999$ \\
\hline \multicolumn{5}{|l|}{ Clinical manifestations at study visit, \% } \\
\hline Tiredness & 66.7 & 50.0 & 79.0 & 0.136 \\
\hline Joint swelling & 54.6 & 42.9 & 63.2 & 0.247 \\
\hline Morning stiffness & 90.9 & 78.6 & 100 & 0.067 \\
\hline HAQ-DI mean score (SD) & $0.95(0.56)$ & $0.63(0.50)$ & $1.19(0.47)$ & 0.006 \\
\hline \multicolumn{5}{|l|}{ HAQ-DI categories, mean score (SD) } \\
\hline Dressing and grooming & $0.8(0.7)$ & $0.4(0.5)$ & $1.2(0.6)$ & $<0.001$ \\
\hline Arising & $1.2(0.7)$ & $0.8(0.7)$ & $1.4(0.5)$ & 0.009 \\
\hline Eating & $0.6(0.7)$ & $0.4(0.6)$ & $0.8(0.8)$ & 0.070 \\
\hline Walking & $1.1(0.7)$ & $0.9(0.7)$ & $1.3(0.7)$ & 0.083 \\
\hline Hygiene & $0.7(0.6)$ & $0.5(0.7)$ & $0.9(0.5)$ & 0.034 \\
\hline Reaching & $1.2(0.9)$ & $0.9(0.9)$ & $1.5(0.8)$ & 0.025 \\
\hline Grip & $0.8(0.8)$ & $0.6(0.6)$ & $1.0(0.8)$ & 0.121 \\
\hline Usual activities & $1.1(0.8)$ & $0.7(0.8)$ & $1.4(0.7)$ & 0.006 \\
\hline
\end{tabular}

$\mathrm{CHIKV}=$ chikungunya virus; HAQ-DI = Health Assessment Questionnaire Disability Index.

with chronic CHIKV arthritis. The disparities observed between our work and other studies could be attributed to diversity in genetic and socioeconomic factors as well as differences in CHIKV genotypes.

Some manifestations on the acute phase have been associated with the development of chronic CHIKV arthritis, but these vary among different ethnic groups. Fever, tiredness, and myalgias at the onset of CHIKV infection were associated with chronic arthritis in our patients. Similarly, Heath et al. ${ }^{29}$ found that fever at initial infection was a risk factor for chronic disease, whereas Murillo-Zamora et al. ${ }^{16}$ found an association with gastrointestinal symptoms such as diarrhea, vomiting, and abdominal pain. Conversely, studies from Brazil and La Martinique did not find a relationship with fever or myalgias at the acute phase. ${ }^{13,19}$

In our patients with chronic CHIK arthritis, the most commonly involved joints were MCPs, shoulders, and PIPs. This finding is in agreement with other studies in which upper extremities are the most affected. ${ }^{19,30}$ By contrast, Rahim et al. ${ }^{11}$ reported predominant lower limb involvement (knees and ankles) over upper limbs (elbows, wrists, and shoulders). The single consensus among all studies of patients with chronic $\mathrm{CHIKV}$ arthritis is the involvement of both small and large joints. Unique to our study is the unaltered clinical expression due to lack of treatment with DMARDs or corticosteroids. Also, most studies evaluated involved joints through surveys frequently administered by telephone. In our study, an expert rheumatologist performed the history and physical examination, eliminating subjective bias from respondents or variability on the assessments from multiple physicians.

As in our work, studies evaluating disease activity in patients with chronic CHIKV arthritis have consistently demonstrated a high disease burden comparable to autoimmune inflammatory arthritides. In studies using DAS28, patients with chronic CHIKV arthritis display moderate-to-high disease activity using the established threshold for RA. For example, Chang et al. $^{31}$ measured the mean (SD) DAS28-C-reactive protein (CRP) in patients with chronic CHIKV arthritis and found a high disease activity $(4.5 \pm 0.8)$. Even after excluding patients with preexisting arthritic disorders, disease activity 
remained high at $4.4 \pm 0.7$. Similarly, Hyad et al. ${ }^{19}$ found a high mean DAS28 score at $4.4 \pm 1.1$. A limitation in their study is that CRP levels were assumed to be normal for all patients because of a lack of laboratories, likely underestimating disease activity scores. In another study using RAPID3 to measure disease activity, $68 \%$ had moderate-to-high disease activity. ${ }^{9}$ As noted before, in these studies, patients treated with DMARDs or that had recent corticosteroid exposures were included. Our study group with chronic CHIKV arthritis could have excluded patients with presumably severe disease that warrant DMARDs therapy. Despite this selection bias, disease activity per CDAl was moderate-to-high in $58 \%$ of our patients.

Not only disease severity was substantial in our patients, but functional disability measured by the HAQ-DI was considerable too. The mean HAQ-DI score in our patients with chronic CHIKV arthritis was $0.95 \pm 0.56$. Furthermore, in those with moderate-to-high disease activity, the mean HAQ-DI score increased to $1.19 \pm 0.47$. These HAQ-DI scores are comparable to patients with RA, osteoarthritis, and fibromyalgia. $^{32-35}$ The HAQ-DI categories in which our patients had more disability were arising, reaching, walking, and performing usual activities. Similar to our study, in patients from South India, the most affected HAQ-DI categories were (in decreasing order) arising, walking, performing usual activities, and reaching for those with a severe disability. ${ }^{11}$ Amaral et al. ${ }^{18}$ reported a mean HAQ-DI score of $1.0 \pm 0.4$ in Brazilian patients with chronic $\mathrm{CHIKV}$ arthritis. In addition, they evaluated five other studies measuring functional status and found a combined HAQ-DI mean score of $1.59 \pm 0.46$. Four of the five studies had a mean HAQ-DI score consistent with moderate-to-severe disability (HAQ-DI $\geq 1) .{ }^{18}$ On the other hand, in the RHEUMATOCHIK study, the mean HAQ-DI score was lower at $0.44 \pm 0.5$. The authors explained that this relatively low score was possibly due to younger age of patients as well as the heterogeneity of CHIKV strains. ${ }^{10}$ In contrast to our study, all these works included patients treated with antimalarial drugs, methotrexate, biologics, and corticosteroids, and occasionally included patients with other rheumatic inflammatory arthritides.

Our study has some limitations. First, because we matched our patients and controls for age and gender, we could not evaluate if these demographic factors were associated with chronic CHIKV. In other studies, age and female gender were found to be risk factors for chronic CHIKV arthritis. ${ }^{8,15,24,28}$ Second, we did not examine additional risk factors for chronic CHIKV arthritis, such as the severity or duration of symptoms during the acute phase and viremia (CHIKV RNA in serum, mean lgG/lgM levels, and/or persistence of lgM). Third, our study was conducted in Puerto Ricans for which the results may not be extrapolated to other ethnic groups. Finally, it would be important to determine further inflammatory markers relevant to establish chronicity. Also, it would be interesting to explore the role of the inflammasome in the initial phase and if HLA-DR4 patterns may orchestrate the chronicity. These two immunogenetic features might have therapeutic implications.

In summary, to the best of our knowledge, this is the first study that determines the clinical manifestations, disease activity, and functional status in patients with chronic CHIKV arthritis who are naive to synthetic or biologic DMARDs. In addition, we excluded patients who received treatment with corticosteroids during the chronic phase and those who had other autoimmune rheumatic diseases. Thus, the findings described here are those of patients with unaltered clinical expression and solely secondary to chronic CHIKV arthritis. In our group of patients, diabetes mellitus, chronic back pain, and manifestations (fever, tiredness, and myalgias) on the acute phase of infection were associated with chronic $\mathrm{CHIKV}$ arthritis. Those with chronic CHIKV arthritis had substantial disease activity and functional disability.

Received December 10, 2020. Accepted for publication January 22, 2021.

Published online March 8, 2021.

Acknowledgments: We are grateful to Luisa Alvarado and Vanessa Rivera-Amill to facilitate the enrollment of CHIKV patients at the Ponce Health Sciences University, Ponce, Puerto Rico.

Financial support: This work was supported by the National Institute of General Medical Sciences (NIGMS) grant number U54GM133807 for the Hispanic Alliance for Clinical and Translational Research and the National Institute of Minority Health and Health Disparities grant number G12MD007600 for the Research Centers for Minority Institutions (RCMI) Program.

Authors' addresses: Nicolle Medina-Cintrón, Yaritza Berríos-López, and Luis M. Vilá, Division of Rheumatology, University of Puerto Rico Medical Sciences Campus, San Juan, Puerto Rico, E-mails: nicolle. medina@upr.edu, yaritza.berrios@upr.edu, and luis.vila2@upr.edu. Idali Martínez, Department of Microbiology and Medical Zoology, University of Puerto Rico Medical Sciences Campus, San Juan, Puerto Rico, E-mail: idali.martinez@upr.edu. Naydi Pérez-Ríos, Hispanic Alliance for Clinical and Translational Research, University of Puerto Rico Medical Sciences Campus, San Juan, Puerto Rico, E-mail: naydi.perez@upr.edu.

\section{REFERENCES}

1. Amaral JK, Taylor PC, Teixeira MM, Morrison TET, Schoen RT, 2019. The clinical features, pathogenesis and methotrexate therapy of chronic chikungunya arthritis. Viruses 11: 289.

2. Suhrbier A, 2019. Rheumatic manifestations of chikungunya: emerging concepts and interventions. Nat Rev Rheumatol 15: 597-611.

3. Robinson MC, 1955. An epidemic of virus disease in southern province, Tanganyika territory, in 1952-1953. I. Clinical features. Trans $R$ Soc Trop Med Hyg 49: 28-32.

4. Monge $P$ et al., 2019. Pan-American league of associations for rheumatology-central American, Caribbean and Andean rheumatology association consensus-conference endorsements and recommendations on the diagnosis and treatment of chikungunya-related inflammatory arthropathies in Latin America. J Clin Rheumatol 25: 101-107.

5. Arroyo-Ávila M, Cabán A, García-Rivera EJ, Irizarry-Pérez M, Torres H, Gorbea H, Vilá LM, 2017. Clinical manifestations associated with peripheral joint involvement in patients with acute chikungunya virus infection. Am J Trop Med Hyg 96: 916-921.

6. Edington F, Varjão D, Melo $P, 2018$. Incidence of articular pain and arthritis after chikungunya fever in the Americas: a systematic review of the literature and meta-analysis. Joint Bone Spine 85: 669-678.

7. Rodríguez-Morales AJ, Cardona-Ospina JA, Fernanda UrbanoGarzón S, Sebastian Hurtado-Zapata J, 2016. Prevalence of post-chikungunya infection chronic inflammatory arthritis: a systematic review and meta-analysis. Arthritis Care Res (Hoboken) 68: 1849-1858.

8. Essackjee K, Goorah S, Ramchurn SK, Cheeneebash J, WalkerBone K, 2013. Prevalence of and risk factors for chronic arthralgia and rheumatoid-like polyarthritis more than 2 years after infection with chikungunya virus. Postgrad Med J: 89: 440-447.

9. Delgado-Enciso l et al., 2018. Smoking and female sex as key risk factors associated with severe arthralgia in acute and chronic phases of Chikungunya virus infection. Exp Ther Med 15: 2634-2642. 
10. Bouquillard E, Fianu A, Bangil M, Charlette N, Ribéra A, Michault A, Favier F, Simon F, Flipo RM, 2018. Rheumatic manifestations associated with Chikungunya virus infection: a study of 307 patients with 32-month follow-up (RHUMATOCHIK study). Joint Bone Spine 85: 207-210.

11. Rahim AA, Thekkekara RJ, Bina T, Paul BJ, 2016. Disability with persistent pain following an epidemic of chikungunya in rural south India. J Rheumatol 43: 440-444.

12. Manimunda SP, Vijayachari $P$, Uppoor R, Sugunan AP, Singh SS, Rai SK, Sudeep AB, Muruganandam N, Chaitanya IK, Guruprasad DR, 2010. Clinical progression of chikungunya fever during acute and chronic arthritic stages and the changes in joint morphology as revealed by imaging. Trans $R$ Soc Trop Med Hyg 104: 392-399.

13. Bertolotti A et al.; Chronic Chikungunya Working Group of University Medical Center of Martinique, 2020. Prevalence of chronic chikungunya and associated risks factors in the French West Indies (La Martinique): a prospective cohort study. PLoS Negl Trop Dis 14: e0007327.

14. Chang AY et al., 2018. Frequency of chronic joint pain following chikungunya virus infection: a Colombian cohort study. Arthritis Rheumatol 70: 578-584.

15. Huits $R$ et al., 2018. Chikungunya virus infection in Aruba: diagnosis, clinical features and predictors of post-chikungunya chronic polyarthralgia. PLoS One 13: e0196630.

16. Murillo-Zamora E, Mendoza-Cano O, Trujillo-Hernández B, Guzmán-Esquivel J, Higareda-Almaraz E, Higareda-Almaraz MA, Sánchez-Piña RA, Lugo-Radillo A, 2018. Persistent arthralgia and related risks factors: a cohort study at 12 months from laboratory-confirmed chikungunya infection. Arch Med Res 49: 65-73.

17. Arroyo-Ávila M, Vilá LM, 2015. Rheumatic manifestations in patients with chikungunya infection. $P$ R Health Sci J 34: 71-77.

18. Amaral JK, Bilsborrow JB, Schoen RT, 2019. Brief report: the disability of chronic chikungunya arthritis. Clin Rheumatol 38: 2011-2014.

19. Hayd RLN, Moreno MR, Naveca F, Amdur R, Suchowiecki K, Watson H, Firestein GS, Simon G, Chang AY, 2020. Persistent chikungunya arthritis in Roraima, Brazil. Clin Rheumatol 39: 2781-2787.

20. Ravindran V, Alias G, 2017. Efficacy of combination DMARD therapy vs. hydroxychloroquine monotherapy in chronic persistent chikungunya arthritis: a 24-week randomized controlled open label study. Clin Rheumatol 36: 1335-1340.

21. Zaid A, Gérardin P, Taylor A, Mostafavi H, Malvy D, Mahalingam S, 2018. Chikungunya arthritis: implications of acute and chronic inflammation mechanisms on disease management. Arthritis Rheumatol 70: 484-495.

22. Aletaha D, Smolen J, 2005. The simplified disease activity index (SDAl) and the clinical disease activity index (CDAl): a review of their usefulness and validity in rheumatoid arthritis. Clin Exp Rheumatol 23: S100-S108.

23. Bruce B, Fries JF, 2003. The stanford health assessment questionnaire (HAQ): a review of its history, issues, progress, and documentation. J Rheumatol 30: 167-178.

24. Schilte C, Staikowsky F, Couderc T, Madec Y, Carpentier F, Kassab S, Albert ML, Lecuit M, Michault A, 2013. Chikungunya virus-associated long-term arthralgia: a 36-month prospective longitudinal study. PLoS Negl Trop Dis 7: e2137.

25. Donath MY, Shoelson SE, 2011. Type 2 diabetes as an inflammatory disease. Nat Rev Immunol 11: 98-107.

26. Teng TS, Kam YW, Lee B, Hapuarachchi HC, Wimal A, Ng LC, Ng $\mathrm{LF}, 2015$. A systematic meta-analysis of immune signatures in patients with acute chikungunya virus infection. $J$ Infect Dis 211: 1925-1935.

27. Chaaitanya IK, Muruganandam N, Sundaram SG, Kawalekar O, Sugunan AP, Manimunda SP, Ghosal SR, Muthumani K, Vijayachari P, 2011. Role of proinflammatory cytokines and chemokines in chronic arthropathy in CHIKV infection. Viral Immunol 24: 265-271.

28. Sissoko D, Malvy D, Ezzedine K, Renault P, Moscetti F, Ledrans M, Pierre V, 2009. Post-epidemic chikungunya disease on Reunion Island: course of rheumatic manifestations and associated factors over a 15-month period. PLoS Negl Trop Dis 3: e389.

29. Heath CJ, Lowther J, Noël TP, Mark-George I, Boothroyd DB, Mitchell G, MacPherson C, Desiree LaBeaud A, 2018. The identification of risk factors for chronic chikungunya arthralgia in Grenada, West Indies: a cross-sectional cohort study. Open Forum Infect Dis 5: ofx234.

30. Amaral JK, Bingham CO, 3rd, Schoen RT, 2020. Successful methotrexate treatment of chronic chikungunya arthritis. J Clin Rheumatol 26: 119-124.

31. Chang AY et al., 2018. Chikungunya arthritis mechanisms in the Americas: a cross-sectional analysis of chikungunya arthritis patients twenty-two months after infection demonstrating no detectable viral persistence in synovial fluid. Arthritis Rheumatol 70: 585-593.

32. Wolfe $F, 2000$. A reappraisal of $H A Q$ disability in rheumatoid arthritis. Arthritis Rheum 43: 2751-2761.

33. Carmona L, Ballina J, Gabriel R, Laffon A; EPISER Study Group, 2001. The burden of musculoskeletal diseases in the general population of Spain: results from a national survey. Ann Rheum Dis 60: 1040-1045.

34. Georgiev T, 2019. Clinical characteristics and disability in patients with knee osteoarthritis: real world experience from Bulgaria. Reumatologia 57: 78-84.

35. Wolfe F, Walitt BT, Katz RS, Häuser W, 2014. Social security work disability and its predictors in patients with fibromyalgia. Arthritis Care Res (Hoboken) 66: 1354-1363. 